\title{
New Statistical Model for Variability of Aerosol Optical Thickness: Theory and Application to MODIS Data over Ocean*
}

\author{
Mikhail D. Alexandrov AND IgOR V. GeOgDZHAYEV \\ Department of Applied Physics and Applied Mathematics, Columbia University, and NASA Goddard Institute for Space Studies, \\ New York, New York \\ KOSTAS TSIGARIDIS \\ Center for Climate Systems Research, Columbia University, and NASA Goddard Institute for Space Studies, New York, New York
}

\author{
ALEXANDER MARSHAK AND ROBERT LEVY \\ NASA Goddard Space Flight Center, Greenbelt, Maryland \\ BRIAN CAIRNS \\ NASA Goddard Institute for Space Studies, New York, New York
}

(Manuscript received 8 May 2015, in final form 6 November 2015)

\begin{abstract}
A novel model for the variability in aerosol optical thickness (AOT) is presented. This model is based on the consideration of AOT fields as realizations of a stochastic process that is the exponent of an underlying Gaussian process with a specific autocorrelation function. In this approach, AOT fields have lognormal PDFs and structure functions with the correct asymptotic behavior at large scales. The latter is an advantage compared with fractal (scale invariant) approaches. The simple analytical form of the structure function in the proposed model facilitates its use for the parameterization of AOT statistics derived from remote sensing data. The new approach is illustrated using a 1-yr-long global MODIS AOT dataset (over ocean) with 10-km resolution. It was used to compute AOT statistics for sample cells forming a grid with $5^{\circ}$ spacing. The observed shapes of the structure functions indicated that, in a large number of cases, the AOT variability is split into two regimes that exhibit different patterns of behavior: small-scale stationary processes and trends reflecting variations at larger scales. The small-scale patterns are suggested to be generated by local aerosols within the marine boundary layer, while the large-scale trends are indicative of elevated aerosols transported from remote continental sources. This assumption is evaluated by comparison of the geographical distributions of these patterns derived from MODIS data with those obtained from the GISS GCM. This study shows considerable potential to enhance comparisons between remote sensing datasets and climate models beyond regional mean AOTs.
\end{abstract}

\section{Introduction}

Atmospheric aerosols through their direct and indirect radiative effects remain a significant source of uncertainty for the historical forcing of climate (Hansen

\footnotetext{
* Supplemental information related to this paper is available at the Journals Online website: http://dx.doi.org/10.1175/JAS-D-15-0130.s1.

Corresponding author address: Mikhail D. Alexandrov, NASA Goddard Institute for Space Studies, 2880 Broadway, New York, NY 10025

E-mail: mda14@columbia.edu
}

et al. 2000; Myhre et al. 2013; Koch et al. 2007; Unger et al. 2008) and consequently for the assessment of projected change. Resolving this uncertainty requires the synergistic combination (through intercomparisons and assimilations) of aerosol models and observational datasets (Kinne et al. 2006; Quaas et al. 2009; Huneeus et al. 2011). As a part of an effort to define new strategies and methodologies for the intercomparison of model and satellite data, it looks promising to include analysis of more detailed characteristics of aerosol variability and go beyond traditional comparison of aerosol optical thickness (AOT) averaged over a geographical region. In particular, structure functions (SFs) provide a 
uniform description of the strength and spatial scale of AOT fluctuations. The structure function [see, e.g., Davis et al. (1994) and the next section] describes the average difference in value over scale. In the framework of traditional scale-invariant (fractal) models, the SF is assumed to have a power-law form characterized by the scaling (Hurst) exponent $H$. SFs together with power spectra have been widely used to characterize scaling of turbulence-driven fluctuations of various atmospheric fields, such as temperature, wind speed, humidity, etc. (e.g., Gage and Nastrom 1986; Lilly 1989; Lovejoy and Schertzer 2010, 2012). Scaling techniques were also successfully used in analysis of various cloud datasets (Cahalan and Snider 1989; Davis et al. 1996, 1997, 1999; Marshak et al. 1997).

The scaling properties of AOT were studied by Anderson et al. (2003) using autocorrelation statistics. This study revealed that mesoscale aerosol variability (at $40-400-\mathrm{km}$ scales) is a common feature of lowertropospheric aerosol light extinction. Another application of scaling analysis to AOT variability was performed by Alexandrov et al. (2004). They studied AOT scaling using the 1-month dataset from a sunphotometer network operated by the U.S. Department of Energy Atmospheric Radiation Measurement Program in Oklahoma and Kansas. The network provided an irregular grid with a mean distance between neighboring sites of roughly $80 \mathrm{~km}$, and the temporal sampling was $20 \mathrm{~s}$. This dataset therefore allowed for both temporal and spatial AOT variability to be analyzed. Alexandrov et al. (2004) found that the temporal variability of AOT can be separated into two scale-invariant regimes: microscale $(0.5-15 \mathrm{~km})$, where fluctuations are governed by $3 \mathrm{D}$ turbulence $(H \approx 0.3)$, and intermediate scale $(15-100 \mathrm{~km})$, characterized by a transition toward large-scale $2 \mathrm{D}$ turbulence $(H \approx 0.4-0.5)$. The temporal evolution of AOT scaling exponents during the month appeared to be correlated with changes in aerosol vertical distribution, while their spatial variability reflected the site's topography.

Unfortunately, the scale-invariant variability model with its power-law SF being divergent at large scales does not naturally reflect an important statistical property of real AOT fields: the statistical independence of AOT values at points separated by a large distance. This property means that the SF approaches a constant value (double the AOT variance) at a sufficiently large scale. To deal with this problem within the fractal framework, a number of scaling regimes are introduced separated by scale breaks. In this study, we present a new AOT variability model that has an advantage over the scaleinvariant approach because its SFs have the correct asymptotic behavior. In this approach, we construct an
AOT field by taking the exponent of an underlying Gaussian random process with a specified autocorrelation function. This ensures that AOT fields have lognormal PDFs (O'Neill et al. 2000), while their structure functions are power law at small scales and approach a constant at large scales. The simple analytical expression for the SF of this model facilitates its application to real AOT datasets.

We will apply our analytical model to the statistics derived from the global Moderate Resolution Imaging Spectroradiometer (MODIS) AOT product (Remer et al. 2005, 2008; Levy et al. 2010). It will be shown that the shapes of the MODIS-derived SFs in many cases suggest the presence of two distinctive variability modes, which we attribute to two aerosol layers separated by height (one within the boundary layer, the other above it). Such a separation adds a "third dimension" to the 2D MODIS dataset and can be quantitatively evaluated by comparison with the aerosol vertical structure in climate models (even if the climate model resolution is insufficient for computation of the SFs themselves). To demonstrate this possibility, we present a comparison between the aerosol modes derived from the MODIS dataset and those obtained using 3D AOT fields simulated by the NASA Goddard Institute for Space Studies (GISS) Model E2 (Schmidt et al. 2014).

\section{Statistics of AOT fields}

Statistical properties of AOT (as well as of many other geophysical parameters) are characterized by their probability distribution (PDF) and structure functions. The latter describes the dependence of the expected difference between AOT values measured at two points in space or time on their separation (see, e.g., Davis et al. 1994; Alexandrov et al. 2004; Lovejoy and Schertzer 2012). The SF is equivalent to the variogram that is used in geostatistics (Curran 1988). In our model, we will use the second-order SF that is defined for a $1 \mathrm{D}$ case as follows:

$$
\begin{aligned}
S_{2}(r) & =\overline{[\tau(x+r)-\tau(x)]^{2}} \\
& =\frac{1}{L-r} \int_{0}^{L-r}[\tau(x+r)-\tau(x)]^{2} d x .
\end{aligned}
$$

Here, $\tau$ is the AOT, $r$ is the lag, or separation between points, and the overbar denotes averaging over $x$ within the range of $[0, L]$, where $L$ is the sample size. This implies the validity of the ergodicity hypothesis such that an ensemble average over realizations is equivalent to an average over the spatial variable $x$. The definition 
of $S_{2}$ is equivalent to that of the variance of the increment field:

$$
\Delta_{r} \tau(x)=\tau(x+r)-\tau(x)
$$

that is,

$$
S_{2}(r)=\operatorname{Var}\left(\Delta_{r} \tau\right)
$$

assuming that $\overline{\Delta_{r} \tau}=0$. The structure function definition for the $2 \mathrm{D}$ case is similar to that for the $1 \mathrm{D}$ case:

$$
S_{2}(r)=\overline{[\tau(\mathbf{x}+\mathbf{r})-\tau(\mathbf{x})]^{2}},
$$

where $\mathbf{x}$ and $\mathbf{r}$ are now $2 \mathrm{D}$ vectors and $r=|\mathbf{r}|$. The averaging in $\mathbf{x}$ is performed over some spatial domain. This implicitly assumes statistical isotropy of the AOT field.

Computation of a structure function (1D or 2D) does not require continuity of the AOT dataset, which can have gaps or even be a collection of values at discrete points in space or time. To derive an SF, we take all available data points and consider all possible pairs of them. For each pair, we calculate the distance and the difference in AOT between the two points. Then the set of these distances and differences from all pairs is used to build a histogram (square difference in AOT vs distance between points), which is the SF for this dataset.

Later in the paper, we will consider a model with two layers, one of which is determined by the marine boundary layer, while the other is advected from elsewhere. Such layers can be regarded as statistically independent. In general, if the aerosol field consists of $n$ statistically independent layers each having an AOT of $\tau^{(i)}(x)$, the total AOT

$$
\tau(x)=\sum_{i=1}^{n} \tau^{(i)}(x)
$$

will have the following statistics:

$$
\bar{\tau}=\sum_{i=1}^{n} \overline{\tau^{(i)}}, \quad \operatorname{Var}(\tau)=\sum_{i=1}^{n} \operatorname{Var}\left[\tau^{(i)}\right]
$$

and

$$
S_{2}(\tau ; r)=\sum_{i=1}^{n} S_{2}\left[\tau^{(i)} ; r\right]
$$

The latter relation follows from Eq. (3).

In the important case of scale-invariant (fractal) fields, structure functions have a power-law form:

$$
S_{2}(r) \propto r^{2 H},
$$

where $H \in(0,1)$ (Mandelbrot 1982). Larger values of $H$ correspond to smoother functions that may have substantial trends, while smaller values indicate finer-scale variability and more stationarity (see, e.g., Marshak et al. 1997). A Hurst exponent of $1 / 2$ corresponds to classical Brownian motion (CBM), which is a Markov process with independent increments. Processes with other values of $H$, called fractional Brownian motions (FBM), are non-Markovian: their increments either correlate (for $H>1 / 2$ ) or anticorrelate (for $H<1 / 2$ ). The theoretical values of $H$ that are characteristic of variability in wind speed and passive scalar advection in turbulent flows are $1 / 3$ for 3D turbulence (Kolmogorov 1941) and 1 for 2D turbulence (e.g., Gage and Nastrom 1986). In their study of sun-photometer-derived AOT time series, Alexandrov et al. (2004) found values of $H$ ranging from 0.1 to 0.6 .

It is known that AOT fields exhibit a scale-invariant structure over certain scale ranges (Alexandrov et al. 2004); however, AOT variability at all scales cannot be described by a single fractal model. It is natural to assume that the AOT values at two points located far enough from each other can be considered independent. Thus, at large scales, $S_{2}(r)$ becomes the variance of the difference between two independent variables, which is equal to the sum of the variances of those variables. As we assume that the AOT field is statistically homogeneous, these variances are the same and equal to the global variance of the dataset. Thus,

$$
S_{2}(r \rightarrow \infty) \simeq 2 \operatorname{Var}(\tau)
$$

is a scale-independent constant. A power-law SF Eq. (8), which diverges at large scales, is inconsistent with this asymptotic constraint (i.e., the global variance does not exist in fractal models). This means that fractal characterization of AOT variability can only be made over a restricted range of scales, and the value of $H$ will be dependent on the range of scales selected. In a model with finite global mean $\bar{\tau}$ and variance $\operatorname{Var}(\tau)=s^{2}$ the autocorrelation function is defined to be

$$
W(r)=\frac{\overline{[\tau(x)-\bar{\tau}][\tau(x+r)-\bar{\tau}]}}{\operatorname{Var}(\tau)},
$$

which is related to the structure function by the expression

$$
S_{2}(r)=2 s^{2}[1-W(r)]
$$

The asymptotic condition Eq. (9) then means that $W \rightarrow 0$ as $r \rightarrow \infty$. 


\section{The statistical model for AOT}

In this section, we will define the AOT variability model and derive the corresponding expressions for structure and autocorrelation functions. AOT datasets are known to have lognormal PDFs (O'Neill et al. 2000), and it is therefore natural to use a statistical model of them where $\tau=\exp (\eta)$, with $\eta$ being a Gaussian field defined by its mean, variance, and autocorrelation function $w(r)$. Realizations of such a process can be constructed using Fourier filtering techniques that use power spectrum computed for a prescribed autocorrelation function (see, e.g., Bell 1987). An alternative method to generate a Gaussian field using the summation of multiple realizations of a binary Markov process is described in the supplemental material.

It is shown in appendix A following the approach described by Mejia and Rodriguez-Iturbe (1974) that the structure and autocorrelation functions of the AOT field can be expressed through the autocorrelation function $w(r)$ of the underlying Gaussian process:

$$
\begin{aligned}
& S_{2}(r)=2 s^{2} \frac{u-u^{w(r)}}{u-1} ; \\
& W(r)=\frac{u^{w(r)}-1}{u-1} .
\end{aligned}
$$

Here, we use the notation

$$
u=\frac{s^{2}+\bar{\tau}^{2}}{\bar{\tau}^{2}}
$$

where $\bar{\tau}$ and $s$ are the mean and standard deviation of the AOT field, respectively. The function $w(r)$ should be positive and obey the following properties: $w(0)=1$ and $w(r \rightarrow \infty)=0$. The former ensures that $S_{2}(0)=0$, while the latter means that $S_{2}(r \rightarrow \infty)=2 s^{2}$. Probably the simplest functional form of $w(r)$ satisfying these conditions is exponential:

$$
w_{M}(r)=e^{-r / L_{e}},
$$

where $L_{e}$ is the autocorrelation length. For example, the autocorrelation function of the Gaussian model based on binary Markov processes (see the supplemental material) has this form. When $w(r)$ is exponential, the structure function is linear in the small-scale limit: $S_{2}\left(r \ll L_{e}\right) \propto r$. This is appropriate for an AOT field that behaves as a classical Brownian motion (having $H=1 / 2$ ). However, in our previous study (Alexandrov et al. 2004) that considered relatively small temporal and spatial scales, we found that the AOT's structure functions showed power-law dependence on the lag: $S_{2}\left(r \ll L_{e}\right) \propto r^{2 H}$. We will see similar small-scale behavior of SFs computed for the MODIS AOT product. This means that, at small scales, real AOT fields resemble FBMs with Hurst exponents not necessarily equal to $1 / 2$. These observations prompt us to generalize the exponential functional shape in Eq. (15) to accommodate the appropriate power-law behavior for the small-scale limit case. We choose the following expression:

$$
w(r)=e^{-\left(r / L_{e}\right)^{2 H}},
$$

which is analytically simple and captures the observed behavior of real AOT fields. For nonexponential $w(r)$, parameter $L_{e}$ is not precisely the autocorrelation length, so we will call it the "characteristic length" instead. It characterizes the typical size of inhomogeneities in the AOT field. Figure 1 shows how the shape of the reduced structure function $S_{2}(r) / 2 s^{2}$ computed according to Eqs. (12) and (16) depends on the three parameters: the relative standard deviation $\nu=s / \bar{\tau}, L_{e}$, and $H$. We see that the dependence on $\nu$ is relatively weak, while variations in $L_{e}$ change the length scale of the function. It is also seen that the SF's value at $r=L_{e}$ does not depend on $H$ (this simplifies fitting of remote sensing data).

\section{Derivation of the model parameters from observations}

We assume that the observational dataset provides a PDF of AOT values (from which we determine $\bar{\tau}$ and $s$ ), as well as the structure function $S_{2}(r)$. Then we compute the parameter $u$ according to Eq. (14) and derive the formula

$$
w(r)=\frac{1}{\ln u} \ln \left[u-\frac{u-1}{2 s^{2}} S_{2}(r)\right]
$$

from Eq. (12). If this function is positive, the model parameters can be obtained from Eq. (16) when it is written as

$$
-\ln w(r)=\left(\frac{r}{L_{e}}\right)^{2 H} .
$$

First, we determine $L_{e}$ from the condition

$$
-\ln w\left(L_{e}\right)=1 .
$$

The small-scale $H$ can then be derived from Eq. (18) by linear regression in $\ln \left(r / L_{e}\right)$ :

$$
\ln [-\ln w(r)]=2 H \ln \left(\frac{r}{L_{e}}\right) .
$$



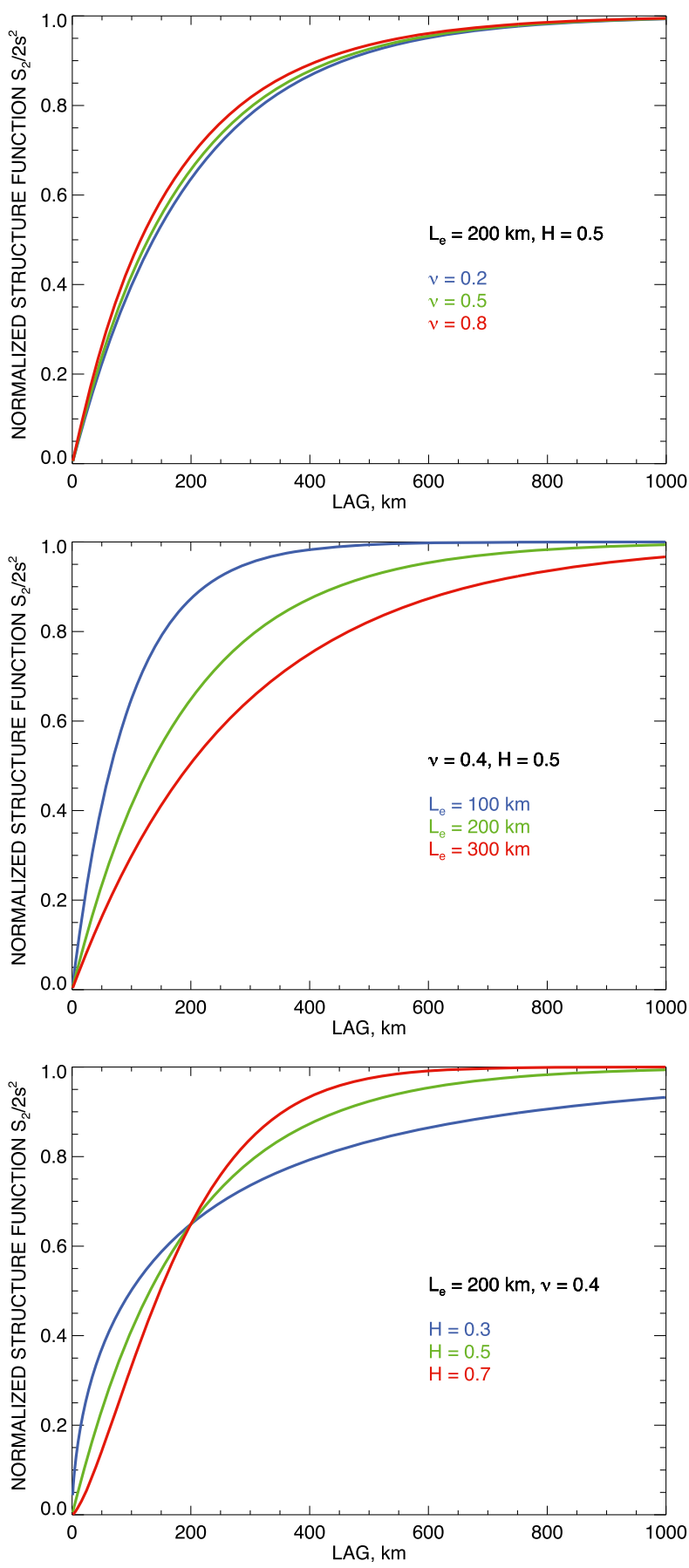

FIG. 1. Dependence of the normalized structure function $S_{2}(r) / 2 s^{2}$ shape [Eqs. (12) and (16)] on the parameters (top) $\nu=s / \bar{\tau}$, (middle) $L_{e}$, and (bottom) $H$.

\section{Application to the MODIS AOT product}

The proposed variability model was evaluated using the AOT product (collection 5, level 2, 550-nm wavelength) from the MODIS instrument on the Terra satellite (Levy et al. 2010). MODIS is on polar orbit, observing a $2330-\mathrm{km}$-wide swath. There are gaps in MODIS observations near the equator, while the measurements from different orbits overlap near the poles. The aerosol retrieval creates a " $10 \mathrm{~km}$ " product, which has $10-\mathrm{km}$ resolution at nadir, extending to $40 \mathrm{~km}$ at swath edge. We took a 1-yr-long (2006) global AOT dataset with $10-\mathrm{km}$ resolution and computed the means, variances, and structure functions for the data from overlapping $10^{\circ} \times 10^{\circ}$ cells (with ocean and land treated separately). The centers of the cells form a grid with $5^{\circ} \times 5^{\circ}$ resolution. To avoid the effects of overlapping orbits on the satellite data at high latitudes, we restricted our retrievals to the area between $60^{\circ} \mathrm{S}$ and $60^{\circ} \mathrm{N}$. Here, we present the results of our analysis only for the measurements over ocean, where variability of surface albedo is small compared to that of AOT.

Computation of structure functions follows the procedure outlined in section 2. For a given day and a given $10^{\circ} \times 10^{\circ}$ cell, we take all available $10 \mathrm{~km} \times$ $10 \mathrm{~km}$ pixels. If the number of these pixels exceeds a threshold of 200 regardless of their distribution within the cell, we proceed with the analysis and consider all possible pairs of pixels. For each pair of pixels, we determine the distance and the difference in AOT between them. After this, we collect these parameters from all pairs and use them to construct a histogram of square difference in AOT versus distance between pixels using a $10-\mathrm{km}$ bin size. This histogram is regarded as the SF for this cell. Note that the computation of SFs for 2D datasets implies statistical isotropy; thus, the resulting structure function is the directionally averaged representation of AOT variability. We estimate the mean and the standard deviation of the AOT in the cell using the data from the available pixels and use these values to parameterize the SF according to our model, as described in section 4 . This procedure, applied to all admissible cells, provides a global daily dataset of $\bar{\tau}, s$, and the SF parameters $L_{e}$ and $H$ on a grid with $5^{\circ}$ resolution (our $10^{\circ} \times 10^{\circ}$ cells overlap). Combining these parameter values over multiple days gives us a time series, which we average over a month or a season (using only the days when the data are available) to obtain the corresponding mean values. The averaging helps to reduce the statistical noise in the dataset. It appears that the above described parameterization does not always provide a good fit to the observed SF because of insufficient sample size (see section 6 for details). While the SF parameters still have a qualitative meaning in such cases, we modify our analysis (as described in section 7) to better explore the information content of the data. 


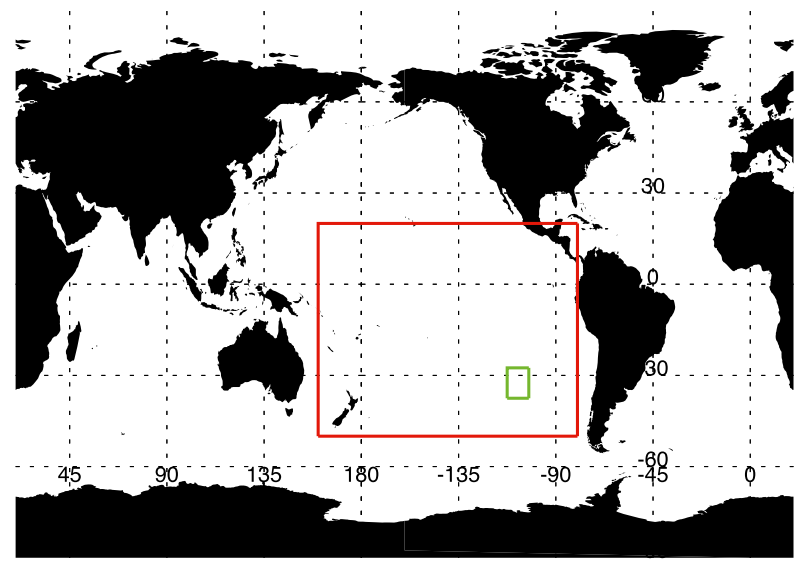

FIG. 2. Two geographical regions of different scales, MODIS data from which were used for computation of structure functions presented in Fig. 3.

\section{Sampling effects}

It is generally difficult to characterize the accuracy of structure functions computed from satellite data, since in each case we have to deal with a single realization of the stochastic process governing the variability in the AOT. The statistics computed using this realization may deviate from those of the (hypothetical) complete statistical ensemble, so we have to assume that this deviation is not significant. Using multiple datasets does not solve this problem since the AOT variability parameters are not the same for different times, locations, and sample sizes. The size of the sample $\left(10^{\circ} \times 10^{\circ}\right.$ in our case) is a free parameter to be chosen by the investigator. It should be large enough to collect enough satellite pixels for statistical analysis, while still sufficiently small to reveal spatial variability in the derived statistics. Another issue with the SF analysis, as well as with any statistical method applied to satellite data, is whether the AOT values at the pixels where retrievals are available are representative of the whole sample area. This is of particular concern if the number of available pixels is small or their spatial distribution is uneven. Note that over ocean, less than $10 \%$ of all global $10-\mathrm{km}$ boxes in the MODIS product have valid AOT retrieval as a result of avoidance of clouds and sun glint. We deal with this issue by setting a threshold on the number of data pixels in the sample and also by controlling the quality of SFs (those that are too noisy to be well fit by our models are discarded).

To give an example of the effect of sample size on the retrieved statistical parameters, we compare structure functions and their parameterizations from two datasets representative of different scales (shown in Fig. 2). One of these areas is a $120^{\circ} \times 70^{\circ}$ region covering more than half of the Pacific Ocean, while the other is its local subset-a typical $10^{\circ} \times 10^{\circ}$ cell used in our analysis. The data are from 17 January 2006. Figure 3 (top left) shows the regional structure function (the AOT data used for the SF computation are shown in the insert). We see that the $5000-\mathrm{km}$ scale is sufficient to observe the beginning of the SF's saturation. The AOT in this sample has a mean of 0.13 and a standard deviation of 0.062 . Despite some noise at larger scales, this SF fits our variability model well with $L_{e}=815 \mathrm{~km}$ and $H=0.39$. Figure 3 (top right) shows the same SF (red curve) over a smaller lag range (up to $1500 \mathrm{~km}$ ) together with the $\mathrm{SF}$ from the $10^{\circ} \times 10^{\circ}$ subset (green curve). We see that, while the local SF largely inherits the shape of the regional one, the $1000-\mathrm{km}$ sampling range is not sufficient to reach the scale at which the SF saturates. Note also that the local standard deviation of 0.042 is $30 \%$ smaller than that for the large region. Figure 3 (bottom) demonstrates that the local SF can be fit by our model in different ways depending on whether the local (Fig. 3, bottom left) or regional (Fig. 3, bottom right) variance value is used, yielding very different values of $L_{e}: 165$ and $845 \mathrm{~km}$, respectively.

Rigorous quantitative estimation of the sampling error in the determination of the SF would have to take into account spatial correlations between the AOT values in different pixels. This is a rather complicated task, which goes beyond the scope of this exploratory study. Instead, we present in Fig. 4 a qualitative analysis of the influence of the number of pixels on the computation of the SF. Figure 4 shows the complete local SF from Fig. 3 (green curve) and the number of pixel pairs $N_{p}$ contributing to this SF at each scale (black curve). This number increases with the lag at small scales, while decreasing at large scales because of the effect of finite sample size. It looks like the effect of $N_{p}$ on the SF starts to dominate once a threshold is crossed. The SF monotonically increases with lag and is in good agreement with its regional analog (Fig. 3, top right) up to the scale of $1100 \mathrm{~km}$ (dashed line in Fig. 4), at which point it breaks. The number of pairs at this lag is 1800 . The reason for this behavior is in the rapid growth at this point of $N_{p}^{-1 / 2}$, which determines the statistical uncertainty of the SF computation. This is illustrated in Fig. 4 by two orange curves corresponding to $S_{2} \pm$ constant $\times N_{p}^{-1 / 2}$ [the constant here is taken equal to $\left.2 \max \left(S_{2}\right)\right]$. The admissible scale range with a number of pairs larger than this value is shown in Fig. 4 by the blue horizontal line. Besides the part with $r>1100 \mathrm{~km}$, this range does not include the first two bins corresponding to lags of less than $20 \mathrm{~km}$. We will see below that such a scale range is sufficient for SF parameterization.

The example described above demonstrates that there are two negative effects of having a smaller sample size 

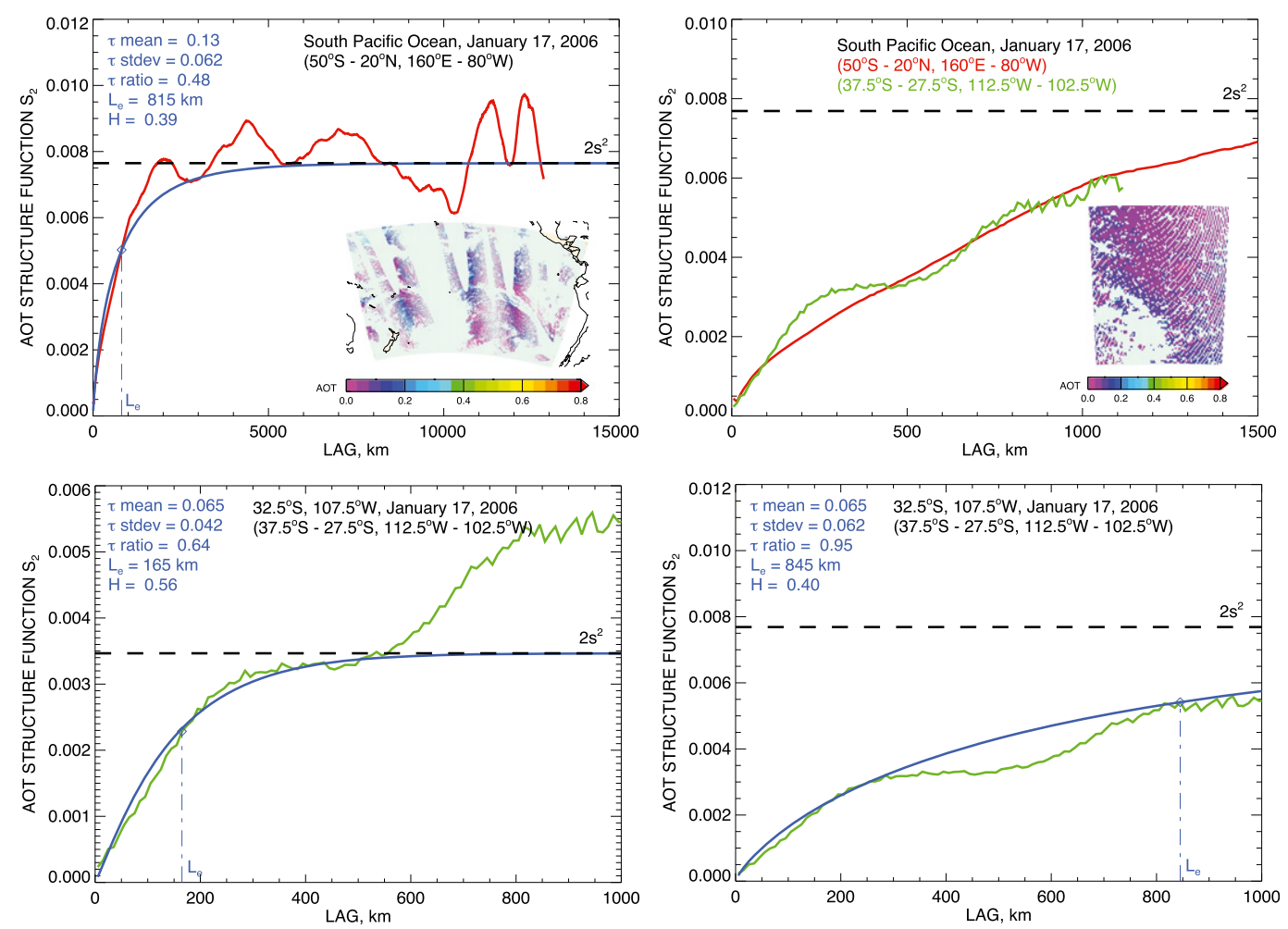

FIG. 3. Structure functions computed from MODIS data over the South Pacific Ocean obtained on 17 Jan 2006. (top left) SF (red) for large $120^{\circ} \times 70^{\circ}$ area exhibiting statistical saturation at large scales. The blue curve represents the parametric fit to the data. (top right) SF (red) for large $120^{\circ} \times 70^{\circ}$ area exhibiting statistical saturation at shorter scale range in comparison with local SF (green) from a $10^{\circ} \times 10^{\circ}$ area. Maps presenting MODIS pixels used for SF computations are included as inserts. (bottom left) Fit to the local SF using locally measured standard deviation. (bottom right) As in (bottom left), but using the large-scale value of the standard deviation from (top left).

on SFs and their parameterizations. First, the reduction in the number of pixel pairs especially affects the largescale range, where the structure function is expected to saturate to its asymptotic value. This may yield incomplete SFs showing no saturation at all. Second, the dependence of the AOT variance on the sample size (when this size is small) may lead to ambiguity in parameterization of SFs.

\section{Information content of incomplete structure functions}

While the example from the previous section shows that an incomplete (not reaching saturation) structure function cannot be used for the retrieval of regionalscale statistics, such SFs can still provide valuable information on AOT variability at specific geographic locations. A closer look at Fig. 3 (top right) reveals a feature in the local SF curve at scales smaller than $600 \mathrm{~km}$ distinguishing it from the regional SF. We call this feature a partial saturation. Figure 3 (bottom left) demonstrates that the shape of the local SF in this scale range is consistent with our model if the local variance is used in the fitting process. The fit yields $H=0.56$ and $L_{e}=165 \mathrm{~km}$. This behavior can be explained by the presence of trends in small samples. These trends reflect the nonstationary nature of AOT variability at small scales (where it behaves as an FBM) and are averaged out in statistics for a sufficiently large dataset. To explain the shape of the partial saturation feature, we decompose (using, e.g., linear regression) the $1 \mathrm{D}$ or $2 \mathrm{D}$ small-scale AOT sample into a sum of two independent components: a trend (which is close to a linear function) and a stationary field. Then, according to Eq. (7), the total SF can be represented as a sum of SFs of these components. By the nature of this decomposition, the stationary component's SF quickly saturates at scales smaller than the typical trend length. The structure function of the trend component has the form $S_{2}(r) \propto r^{2}$ (corresponding to fractal model with $H=1$ ). The plot of a sum of such two functions (see Fig. 5) is similar to those in Figs. 3 and 6. Here, we see a partial saturation at 


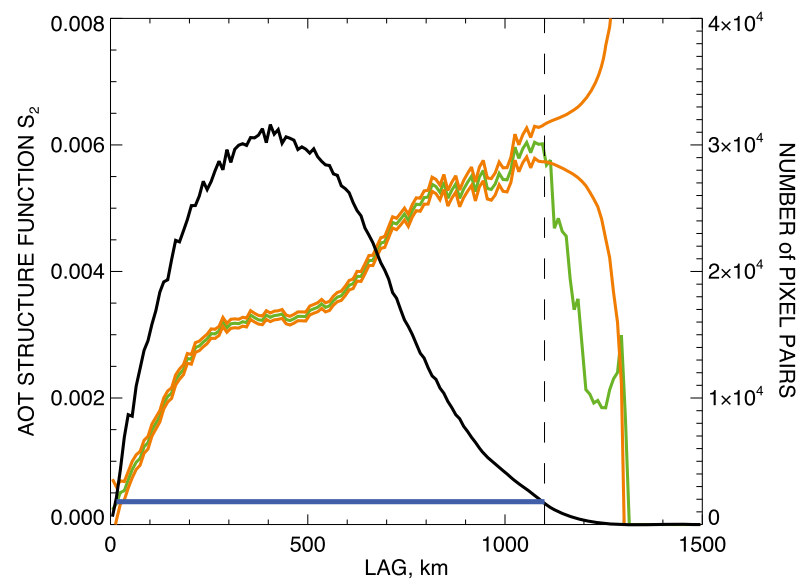

FIG. 4. Structure function from Fig. 3 (green) and the number of MODIS pixel pairs $N_{p}$ that contributed to the SF value at each lag (black). The lag interval with admissible number of pairs $(>1800)$ is indicated by the blue horizontal line. The two orange curves corresponding to $S_{2} \pm$ constant $\times N_{p}^{-1 / 2}$ illustrate the growth of SF computation uncertainty with the decline of $N_{p}$.

smaller scales (inherited from the stationary SF) followed by an increase at larger scales where the trend's SF starts to dominate. This pattern does not affect scales larger than the typical length of a trend.

The strength of a trend can be evaluated by the difference between the AOT variance in the sample and the stationary component variance inferred from the saturation value of its SF. For example, we see that the trend contributions to the SFs in Figs. 3 (bottom left) and $6 a$ are weak, since in these cases the AOT variances in the samples can be explained by the stationary components alone. On the other hand, the SFs in Figs. 6b-e show indications of stronger trends.

While some trends can be present in an ambient aerosol layer (as is likely to be the case in Fig. 3), stronger trends may indicate the presence of aerosol plumes transported above the marine boundary layer (MBL) from remote continental sources. Such plumes are large in scale and relatively smooth, since they are not affected by boundary layer turbulence. They are also localized (being a plume) by proximity to their sources and characteristic wind patterns. This localization induces trends in AOT between the center of the plume and its edges. This allows us to assume a two-mode aerosol structure with a transported mode located above the MBL and associated with the trend component in AOT and a local (or MBL) mode located within the MBL and associated with the stationary component in AOT.

The parameters of the transported-mode SF cannot be retrieved using the local AOT variance; however, the MBL-mode SF can be separated and characterized fairly

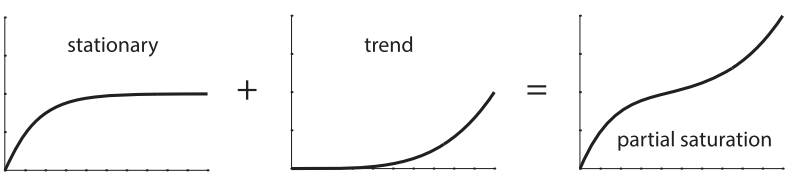

FIG. 5. Schematic representation of the partial saturation feature seen in structure functions from Figs. 6 and 3 as a sum of stationary and trend components.

well because of its small-scale saturation (see appendix $\mathrm{B}$ for a description of the technique). Knowing the mean and the variance of the MBL AOT allows us to also determine the parameters of the transported mode by subtraction of the MBL values from those of the total SF.

Besides the two-mode method, we also continue to employ the single-mode technique described in section 4. It uses the local variance and derives the values of $L_{e}$ and $H$. While the quality of the fit of the SF to a singlemode model may be less than perfect, the characteristic lengths $L_{e}$ obtained in this way still provide a proxy for the scale of total (not just MBL) AOT variability.

\section{Examples of structure functions from MODIS dataset}

Figure 6 presents examples of the structure functions computed using MODIS data from five different $10^{\circ} \times$ $10^{\circ}$ ocean regions (shown in Fig. 6, top left). All the data are from the same day, 18 August 2006, and the pixels used are shown in the inserts. In three out of five of the presented cases, SFs show pronounced partial saturation at scales below $400 \mathrm{~km}$ indicative of strong trends in AOT. One of the exceptions is the case from the relatively pristine Pacific Ocean that is unaffected by longrange aerosol transport (Fig. 6a; similar to Fig. 3, bottom left). The absence of a trend contribution to the AOT variance and the short characteristic length of the MBL component $\left(L_{e}=65 \mathrm{~km}\right)$ suggest that the aerosol in this area is predominantly from local sources (e.g., sea spray). The SF from African coastal waters (Fig. 6b) looks quite different. A large AOT value (1.0) and partial saturation in the SF are consistent with significant amounts of Saharan dust in this region. There the aerosol has essentially a two-layer structure with a lower local aerosol (e.g., sea spray) within the marine boundary layer (which typically has a height of 500-600 m) and an elevated dust layer transported from continental sources at $2-5 \mathrm{~km}$ above the sea level. If we assume that $\tau \propto s$, then two-thirds of AOT in this case comes from the elevated layer. The single-mode estimate of the variability scale is large $\left(L_{e}=475 \mathrm{~km}\right)$, while the MBL component's SF has a much more modest scale $L_{e}=115 \mathrm{~km}$. Besides locally produced sea spray, the 

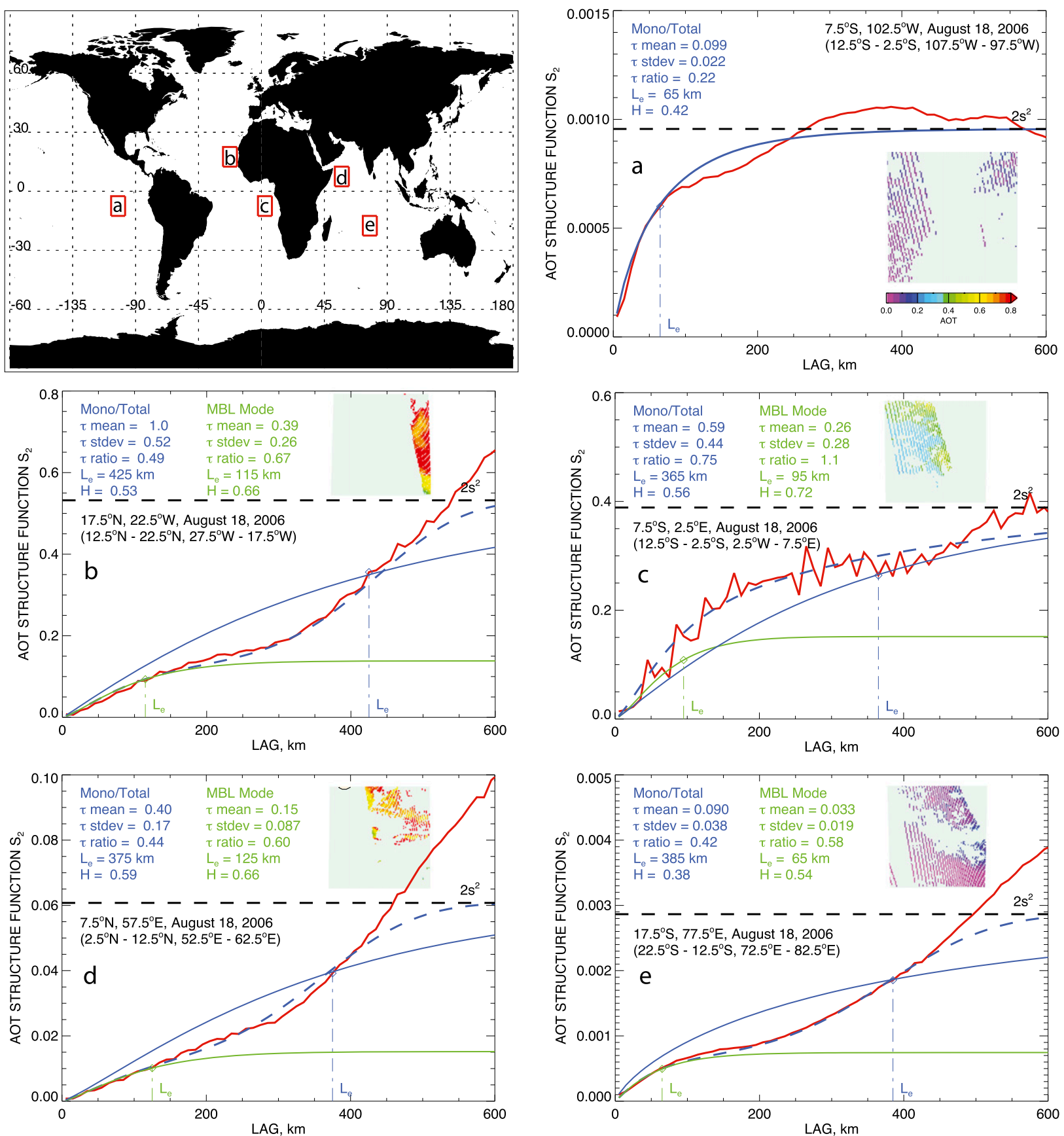

FIG. 6. Structure functions computed using MODIS data from various $10^{\circ} \times 10^{\circ}$ ocean regions obtained on 18 Aug 2006 . (top left) The lettered regions correspond with (a) relatively pristine Pacific Ocean unaffected by long-range aerosol transport; (b) African coastal waters with strong presence of Saharan dust; (c) area off coast of equatorial Africa affected by biomass-burning smoke; (d) northern Indian Ocean with presence of dust from Arabian Peninsula; and (e) middle of the Indian Ocean, data that still show presence of the transported aerosol. In (a)-(e), the red curve corresponds to the SF derived from the data, while the fits obtained from single-mode (solid) and two-mode (dashed) models are shown by blue lines. The SFs and parameters of the stationary MBL AOT component are shown in green. Maps showing the actual MODIS pixels used for SF computations are included in (a)-(e) as inserts.

MBL can also contain some dust falling from the elevated layer. Figure $6 \mathrm{c}$ presents the SF from an area off the coast of equatorial Africa that is known to be affected by biomass burning smoke from the continent.
While this is quite an interesting region to study, the data look consistently noisier than those from other places. The relatively large ratio $s / \bar{\tau}=0.75$ indicates intermittency (presence of isolated high values) in the 
sample and may point to undetected clouds below the smoke layer. Some inconsistency between MODIS and CALIOP AOT from this region and season was also reported by Redemann et al. (2012). Figure $6 d$ shows the $\mathrm{SF}$ from the northern Indian Ocean off the coast of the Somali Peninsula. This area is affected by dust transport from the Arabian Peninsula. This structure function looks similar to that for the Saharan dust case (Fig. 6b) and has similar parameters; however, the AOT here is much smaller: 0.4. The SF from the middle of the Indian Ocean (Fig. 6e) also has a pronounced partial saturation feature, while the AOT $\approx 0.1$ there is as small as in the Pacific Ocean case. This may indicate the presence of a rather thin elevated aerosol layer transported by the west winds (which are strong in this area in summer) from the southern part of Africa.

\section{Geographical mapping of AOT variability}

The parameters of the structure functions derived from the MODIS global satellite dataset together with the means and variances of the AOT can be used to characterize aerosol variability on a planetary scale. We illustrate this possibility by constructing $5^{\circ} \times 5^{\circ}$ resolution maps of the AOT variability parameters averaged over summer (June-August) 2006. Figure 7 presents maps of the mean AOT, its standard deviation, and the ratio of the standard deviation to the mean. It is interesting to observe that this ratio lacks features associated with high-AOT areas (such as Saharan dust or biomass burning smoke), and the whole range of $s / \bar{\tau}$ variability is quite narrow: between 0.3 and 0.6 .

The SF parameters $L_{e}$ and $H$ derived using the singlemode approach are presented in Fig. 8. The larger values of both of these parameters, especially $L_{e}$, correspond to the areas where continental aerosols are advected over the ocean: Saharan dust to the west of northern Africa and biomass burning smoke to the west of the subequatorial part of this continent, dust from the Arabian Peninsula spreading into the northern Indian Ocean, and also smoke and pollution transport from South America, Africa, and Australia driven by the westerly winds of the Southern Ocean (especially in the southern Indian Ocean). Except for the latter case, these areas are associated with large AOT values (see Fig. 7, top). One can observe an unusual low-value feature in the plot of $H$ going in a south-north direction in the western Pacific, close to the international date line. This artifact is probably caused by a known minor problem with the definition of the MODIS day (sometimes an orbit crossing the date line is counted on the wrong day) and can also be seen in some other datasets (see, e.g., Redemann et al. 2012).
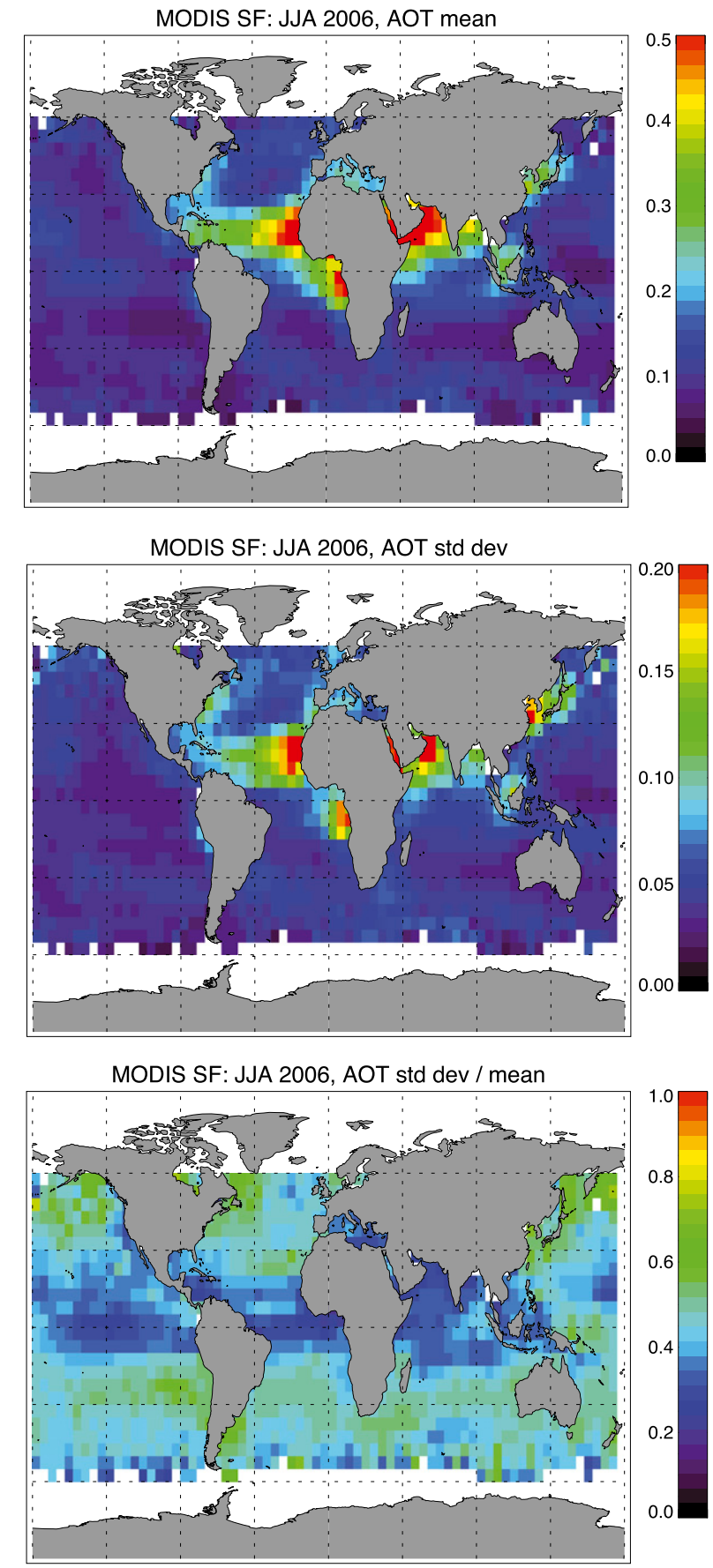

FIG. 7. Seasonal averages of MODIS AOT retrievals for summer 2006: (top) mean, (middle) standard deviation, and (bottom) ratio of the standard deviation and mean.

Under certain assumptions (described in section 7 and in appendix B), the structure function parameterization allows us to split the total AOT into the elevated (transported) and the MBL modes. While the structure function of the elevated mode cannot be reliably characterized, the MBL component's SF can be extracted 

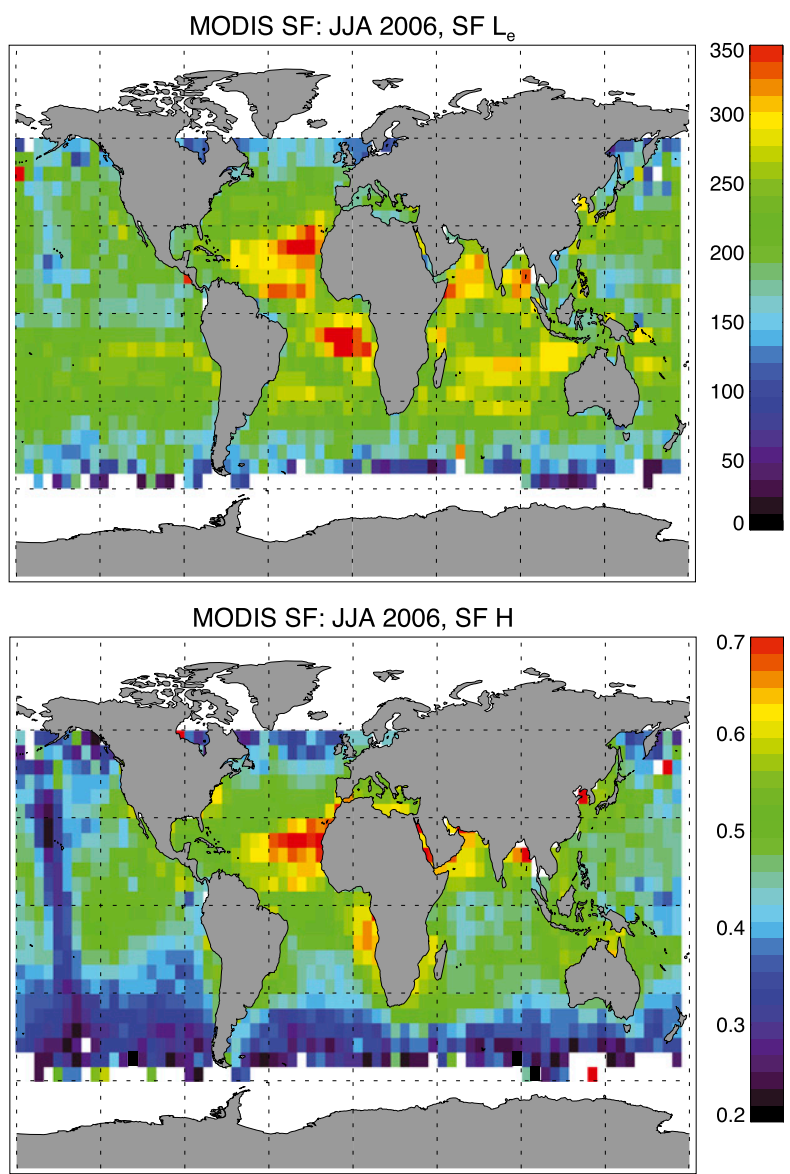

FIG. 8. As in Figs. 7a,b, but for retrieved structure function parameters of single-component model: (top) $L_{e}$ and (bottom) $H$.

and fitted by our model. The typical values of $L_{e}$ for this component are around $100 \mathrm{~km}$ and show little geographical structure, while the exponent $H$ varies between 0.2 and 0.5 with some decrease toward the Southern Ocean. In some cases (such as the one presented in Fig. 6a) only one mode is detected in the SF. In our computations of the averages, we attribute such single-mode AOTs to the elevated mode if $L_{e}>150 \mathrm{~km}$ and to the MBL mode otherwise. The geographical distributions of the AOT components will be discussed in section 10 in comparison with GCM output.

\section{Comparison with GCM output}

While producing a plausible qualitative picture, the proposed layer separation technique needs to be evaluated by comparison with $3 \mathrm{D}$ aerosol datasets. We obtained such a dataset from the NASA GISS GCM simulations for the year 2006. Here, we present comparisons for summer 2006 used in examples of MODIS data described above, while comparisons for all seasons
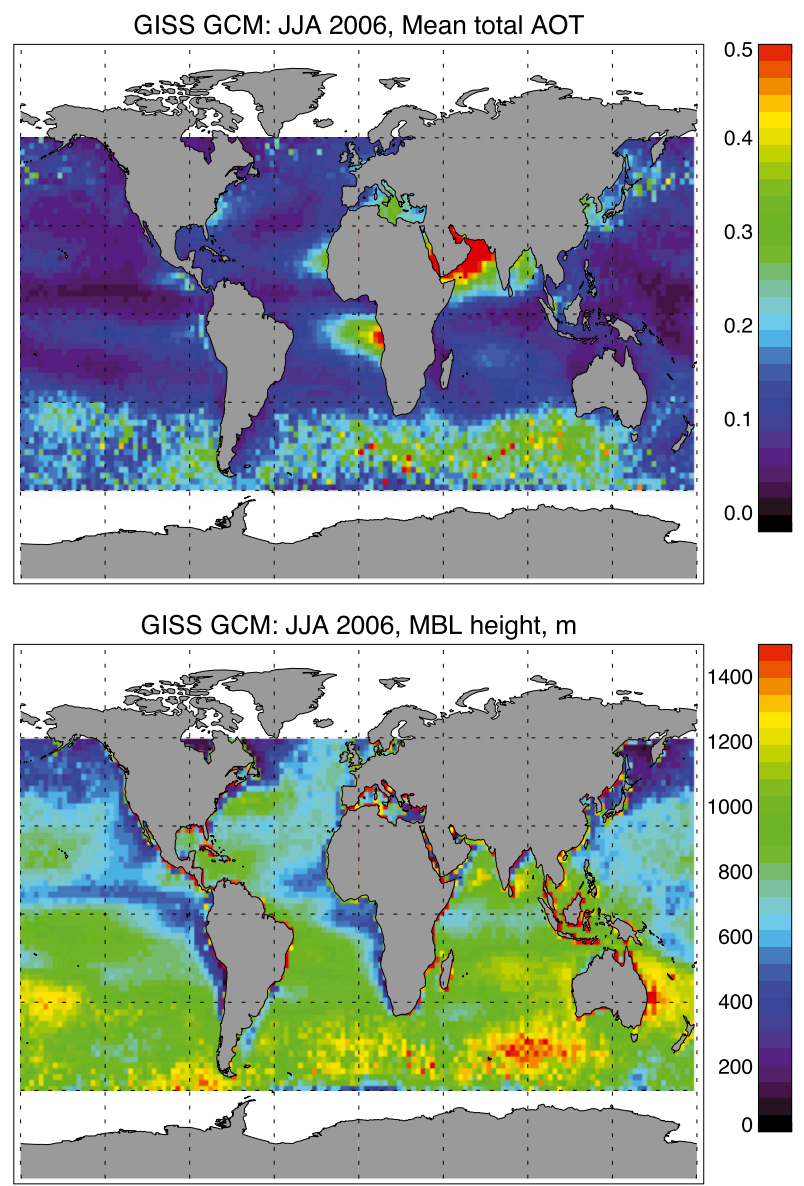

FIG. 9. Maps of seasonal averages from GISS GCM output: (top) total AOT and (bottom) MBL height. The simulations are for summer 2006.

of this year are available as supplemental material to this paper. The GISS Model E2 (Schmidt et al. 2014) produces 3D AOT fields with $2^{\circ}$ resolution in latitude and $2.5^{\circ}$ in longitude. Vertical resolution of the model is defined in sigma units, so it varies with surface pressure. Converted to a height, it is about $200 \mathrm{~m}$ at sea level and increases with altitude. The simulated AOT is divided between several aerosol species: dust, sea salt, biomass burning, industrial pollution, and secondary organic aerosols. Calculation of the boundary layer height in the model is based on the Richardson number criterion, as described by Yao and Cheng (2012).

The seasonally averaged mean AOT map from the GCM simulations is presented in Fig. 9 (top). Visual comparison between this map and that in Fig. 7 (top) reveals a number of qualitative deviations of the model results from the observations, including a notable lack of Saharan dust and a smaller AOT in the Caribbean. The largest model-satellite differences are seen in the Southern Ocean, which is known to be one of the most 
MODIS SF: JJA 2006, Transported mode AOT

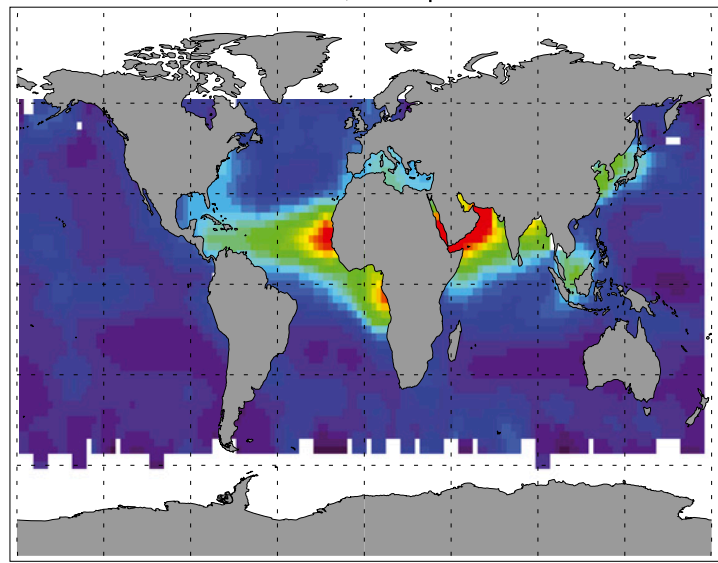

MODIS SF: JJA 2006, MBL mode AOT

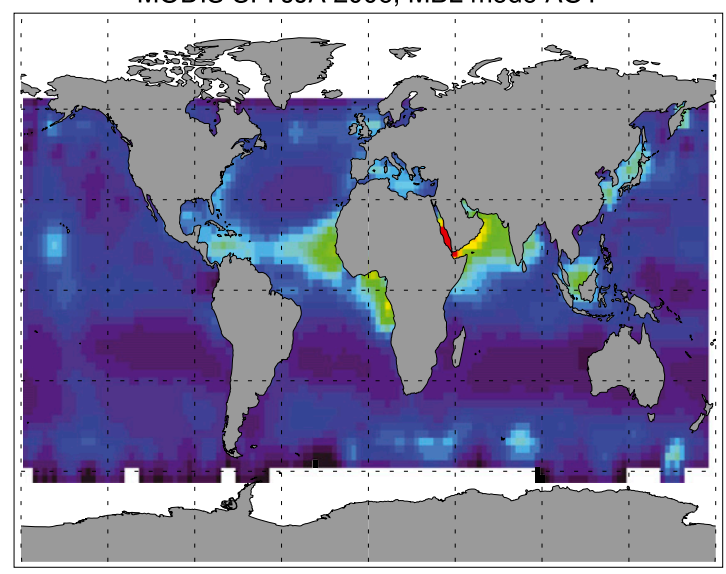

GISS GCM: JJA 2006, Transported mode AOT
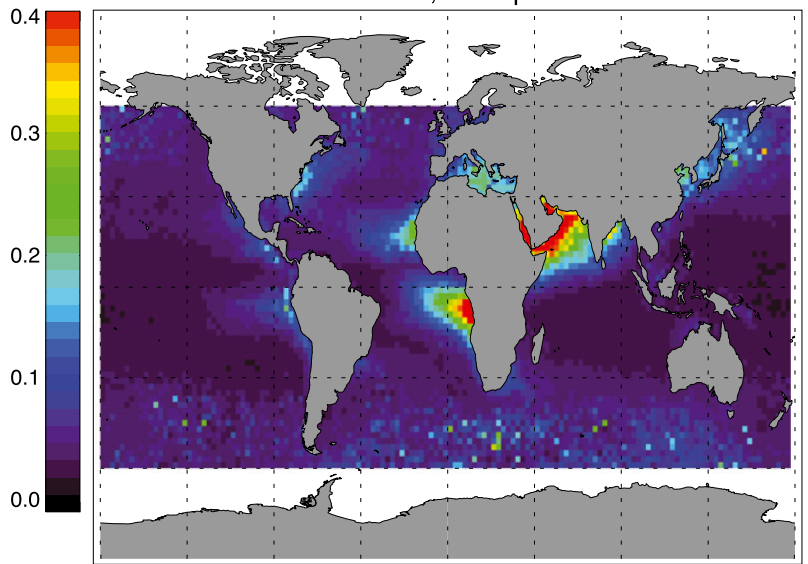

0.4
0.3
0.2
0.1
0.0

FIG. 10. Maps of averaged AOTs for summer 2006: (top) transported and (bottom) MBL components from (left) MODIS structure function analysis and (right) GISS GCM output. The data presented in (left) have been enhanced for comparison with the GCM by interpolating to a $2.5^{\circ} \times 2.5^{\circ}$ grid and smoothing by a moving average. Note that the averages for each mode in MODIS SF dataset were taken only over the days when this mode was present; thus, the component means do not add up to the total mean in Fig. 7 (top).

difficult regions on the planet for both observations and modeling. There the GCM-produced AOT values are as high as $0.3-0.4$, while MODIS detects only background aerosols with an optical thickness of 0.1 or less. These discrepancies in AOT can be caused by many factors, detailed analysis of which is outside the scope of this study. For example, the satellite retrievals can be affected by inadequate cloud screening, while the AOT in the GCM may be biased by an anomalously large MBL height in the Southern Ocean (clearly seen in Fig. 9, bottom), as well as by uncertainties in the assumed size distributions and hygroscopic properties of sea salt aerosols.

Figure 10 presents the partition of the total AOT into the above-MBL (Fig. 10, top) and within-MBL (Fig. 10, bottom) components. Figure 10 (left) shows the results from MODIS SF analysis, while Fig. 10 (right) presents the AOTs obtained from the partition of the GCM AOT profiles by the MBL height (Fig. 9, bottom). Note that the MODIS-derived maps in Fig. 10 were enhanced to make them look similar to the GCM plots: MODIS AOT was interpolated from the original $5^{\circ} \times 5^{\circ}$ grid (as in Fig. 7), to the $2.5^{\circ} \times 2.5^{\circ}$ grid (similar to that of the model) and then smoothed using a moving average. The larger model-satellite differences in the component optical thicknesses are seen in the same regions as those in the total AOTs [e.g., in the Southern Ocean (where the model attributes most of the AOT to the MBL component)]. As to the mode separation in general, we see that in the regions with long-range transport of large aerosol masses (Saharan dust, biomass burning in western Africa) the SF analysis of the MODIS data shows more aerosol in the boundary layer than the model.

The partition of the AOT into two modes allows us to compute the fraction of the total AOT that is in the 
MODIS SF: JJA 2006, Transported mode fraction in AOT

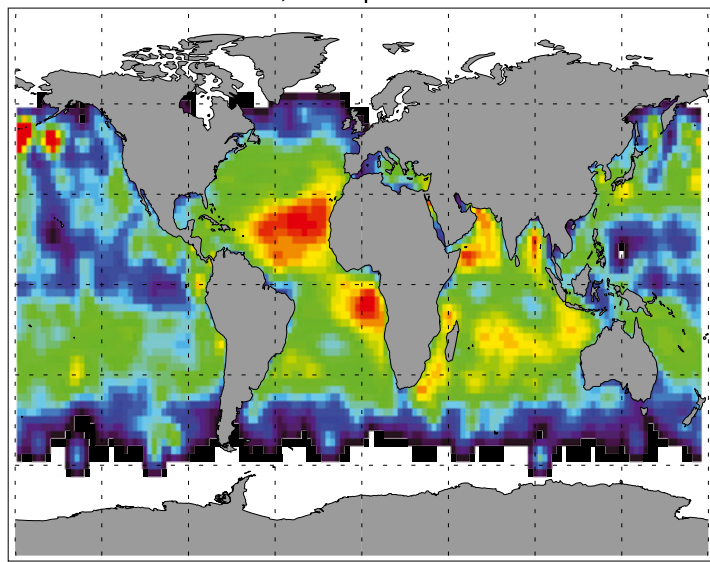

GISS GCM: JJA 2006, Transported mode fraction in AOT
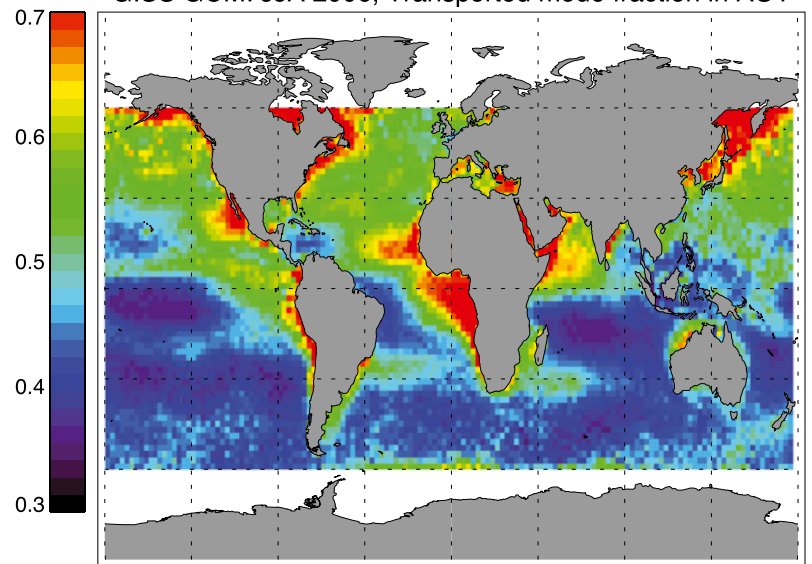

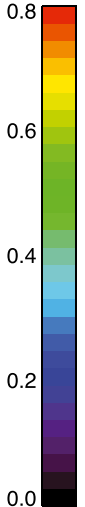

FIG. 11. As in Fig. 10 (top), but for the fractions of the above-MBL AOT in the total AOT. Note that the color bars for (left) MODIS and (right) GISS GCM are chosen differently to enhance comparison of geographical features.

elevated mode. This parameter can be used as an indicator of long-range transport, even when the transported aerosol mass is optically thin. Figure 11 presents the geographical distributions of this ratio derived from the observations (Fig. 11, left) and from the GCM data (Fig. 11, right). Above, we mentioned that mixing between aerosol layers in the model is weaker than in the SF analysis. This means that the GCM AOT ratio plot has more contrast than its MODIS analog: large AOT ratios are larger, while the small ones are smaller. Thus, in order to enhance comparisons between aerosol transport features in the model and observations, we increased the contrast in Fig. 11 (left) by reducing the color range.

The geographical distribution of the AOT ratio in Fig. 11 (left) is similar to that of $L_{e}$ in the single-mode approach that is shown in Fig. 8 (top). This confirms that large-scale features in AOT are associated with elevated plumes. In addition to the West African biomass burning and the dust advected from the Sahara and the Arabian Peninsula, Fig. 11 shows some more subtle similarities that are not readily seen in the total AOT plots. The advection of industrial pollution and biomass burning aerosols from the eastern coasts of Brazil and especially South Africa (across, respectively, the Atlantic and the Indian Oceans) shows stronger presence in observational data than in the GCM results, although it can be seen in both datasets. The same can be said about the feature off the northwestern coast of Australia having very small AOT $(<0.1)$, which is probably associated with smoke. We should note, however, that the quality of the aerosol-mode separation at such small AOTs may be questionable because of the limited accuracy of MODIS retrievals (cf. Levy et al. 2010). The largest differences between the GCM results and our retrievals are seen in the South Pacific, where our interpretation of MODIS data indicates long-range aerosol transport by the westerly winds. However, the model, as it can be seen in Fig. 10, attributes most of the aerosol there to the boundary layer. Relatively high elevated-mode fractions indicate industrial pollution transport from North America and East Asia in both datasets; however, this indication is stronger in the GCM output.

\section{Conclusions}

We introduced a new statistical model for variability of atmospheric AOT. It is based on a representation of AOT fields as realizations of a stochastic process that is the exponent of an underlying Gaussian process with an autocorrelation function of the form given in Eq. (16). The AOT in this model has a lognormal PDF with the mean $\bar{\tau}$ and the standard deviation $s$, while its structure function has the analytical form defined by Eq. (12) with two parameters: the characteristic length $L_{e}$ and the scaling exponent $H$. The AOT fields obeying our model formulation are similar to a fractional Brownian walk with the Hurst exponent $H$ at small scales $\left(r \ll L_{e}\right)$, while they become stationary at large scales $\left(r \gg L_{e}\right)$. This behavior is reflected in the shape of the SF: it has a power-law form at small lags $r$, while approaching a constant in the large-scale limit. This constant is equal to double the AOT's variance, indicating, as expected, that AOT values from distant points are statistically independent. This asymptotic behavior of the SF gives our model an advantage compared to the traditional fractal (scale invariant) model, in which the structure function has a power-law form at any scale, thus diverging in the asymptotic regime. In the fractal framework, variability 
description for a realistic field often requires an artificial split of the scale range into several parts equipped with different fractal models and separated by scale breaks.

The simple analytical form of the SF in our model facilitates its use for parameterization of AOT statistics derived from remote sensing data. We gave examples of such applications using the MODIS AOT product (over ocean) at $10-\mathrm{km}$ spatial resolution. We demonstrated using the data from the $120^{\circ} \times 70^{\circ}$ area in the Pacific Ocean that our statistical model adequately describes AOT variability on a regional scale with SF saturation occurring around 5000-km lag (Fig. 3, top left). We also computed the means, standard deviations, and SFs of the AOT field for seasonal global datasets consisting of overlapping $10^{\circ} \times 10^{\circ}$ sample cells, centers of which form a grid with $5^{\circ}$ spacing. Examples of SFs from a variety of such samples are presented in Fig. 6. While $10^{\circ} \times 10^{\circ}$ or higher grid resolution is necessary to capture geographical differences in the variability patterns of AOT, this sample size appears to be too small for saturation of the SF to be observed. This, together with scaling of the AOT's variance, prevents us from performing a complete parameterization of these structure functions over the whole available scale range. However, some important information on AOT variability can still be obtained from these SFs based on their behavior at scales below $400 \mathrm{~km}$, where they often exhibit partial saturation. This feature is indicative of a split in variability between nonstationary trends and stationary components that we attribute to local processes. The partial SF describing the stationary component saturates at scales around $100 \mathrm{~km}$, so it can be extracted and parameterized according to our model. The presence of a strong trend in the data (that may be associated with long-range transport) can be detected even qualitatively, simply by looking at the shape of the SF. In such a case, the variance corresponding to the partial saturation value of the SF is significantly smaller than the total variance in the sample.

While, rigorously speaking, we only observe the split in the total column AOT variability rather than that in aerosol mass, we can formally associate the large- and small-scale variability patterns with two aerosol modes each having its own fraction of the total AOT. One of these modes corresponds to locally produced aerosol located within the marine boundary layer, while the other represents nonlocal aerosol processes, such as long-range transport above the MBL. Geographical mapping of the results presented in Fig. 8 and Figs. 10 and 11 confirmed that areas where larger values of characteristic lengths and higher fraction of elevatedmode AOT are observed are also known to be affected by long-range aerosol transport (desert dust, biomass burning smoke, etc.). The advantage of our method is in its ability to detect transport of relatively thin aerosol plumes that are not clearly identified in the total AOT datasets.

The set of variability parameters that can be derived from satellite data in addition to the mean AOT has the potential to enhance comparisons between remote sensing datasets and climate models. High-spatialresolution models can now provide data for structure function analysis. Even when a climate model does not have spatial resolution sufficiently high for computation of structure functions, it can still be used to calculate the elevated-mode fraction in AOT, which is comparable to that obtained from SF analysis of satellite data. Indeed, the 3D AOT from a climate model can be divided using the boundary layer height into the MBL and elevated components. In this study, we presented a qualitative example of such a comparison between AOT mode separation results from MODIS SF analysis and from the GISS GCM simulations. Despite some differences described in section 10, both datasets showed many similar aerosol transport patterns. Such comparisons are very useful for further development and testing of the SF technique and also for evaluating and improving the models, especially in terms of their long-range transport and aerosol lifetime.

We plan to continue such comparisons in the future, also involving aerosol-height-resolved measurements, such as those made by Cloud-Aerosol Lidar with Orthogonal Polarization (CALIOP) onboard the NASA Cloud-Aerosol Lidar and Infrared Pathfinder Satellite Observations (CALIPSO) satellite (Winker et al. 2009, 2010), as well as high-resolution GCMs. For example, our preliminary tests showed that the $1.125^{\circ} \times 1.125^{\circ}$ resolution of the European Centre for Medium-Range Weather Forecasts (ECMWF) model (Morcrette et al. 2009; Benedetti et al. 2009) or the Spectral RadiationTransport Model for Aerosol Species (SPRINTARS) (Takemura et al. 2000, 2005; Geogdzhayev et al. 2014) is sufficient for the computation of structure functions for $10^{\circ} \times 10^{\circ}$ samples.

Acknowledgments. This research was funded by the NASA Radiation Sciences Program managed by Hal Maring. Kostas Tsigaridis acknowledges support from NASA's Atmospheric Composition Modeling and Analysis Program (ACMAP), Contract NNX15AE36G. Resources supporting this work were provided by the NASA High-End Computing (HEC) Program through the NASA Center for Climate Simulation (NCCS) at Goddard Space Flight Center. We thank the two anonymous reviewers for thoughtful remarks that allowed us to substantially improve the paper. We also thank A. Davis and M. Mishchenko for useful discussions. 


\section{APPENDIX A}

\section{Statistics of Modeled AOT Fields}

Here, we derive the statistics of the exponential AOT field

$$
\tau=e^{\eta}
$$

based on a Gaussian process $\eta$ having the mean $\mu$, variance $\sigma^{2}$, and autocorrelation function $w(r)$. The field $\tau$ has lognormal PDF with the mean

$$
\bar{\tau}=e^{\mu+\sigma^{2} / 2}
$$

and the variance

$$
s^{2}=\left(e^{\sigma^{2}}-1\right) \bar{\tau}^{2}=(u-1) \bar{\tau}^{2} .
$$

Here, we introduced the parameter

$$
u=e^{\sigma^{2}}=\frac{s^{2}+\bar{\tau}^{2}}{\bar{\tau}^{2}} .
$$

We start derivation of the structure function for $\tau$ with computation of the corresponding autocorrelation function. The covariance between $\tau_{1}=\tau(t)$ and $\tau_{2}=\tau(t+r)$ has the form

$$
\operatorname{Cov}\left(\tau_{1}, \tau_{2}\right)=\overline{\left(\tau_{1}-\bar{\tau}\right)\left(\tau_{2}-\bar{\tau}\right)}=\overline{\tau_{1} \tau_{2}}-\bar{\tau}^{2} .
$$

To compute it, we need to know the mean of $\tau_{1} \tau_{2}=\exp \left(\eta_{1}+\eta_{2}\right)$. The random variable $\eta_{1}+\eta_{2}$ being a sum of normally distributed variables is normally distributed itself. It has the mean $2 \mu$ and the variance

$$
\begin{aligned}
\operatorname{Var}\left(\eta_{1}+\eta_{2}\right) & =2 \operatorname{Var}(\eta)+2 \operatorname{Cov}\left(\eta_{1}+\eta_{2}\right) \\
& =2 \sigma^{2}[1+w(r)] .
\end{aligned}
$$

Thus, the exponent of this variable is distributed lognormally with the mean

$$
\overline{\tau_{2} \tau_{1}}=e^{2 \mu+\sigma^{2}(1+w)}=\bar{\tau}^{2} u^{w},
$$

and the autocorrelation function for $\tau$ has the form

$$
W(r)=\frac{\bar{\tau}^{2}\left[u^{w(r)}-1\right]}{s^{2}}=\frac{u^{w(r)}-1}{u-1} .
$$

Note that, as $w(0)=1$ and $w(r \rightarrow \infty)=0, W(r)$ has the same properties. The structure function can be computed according to Eq. (11):

$$
S_{2}(r)=2 s^{2} \frac{u-u^{w(r)}}{u-1}=2 s^{2} \frac{u}{u-1}\left[1-u^{-z(r)}\right],
$$

where $z(r)=1-w(r)$. It is easy to see that $S(0)=0$ and $S_{2}(r \rightarrow \infty)=2 s^{2}$. In the small-scale limit, if we assume $z(r) \propto r^{2 H}$, the structure function has the same powerlaw behavior:

$$
S_{2}(r \rightarrow 0) \propto r^{2 H},
$$

indicating that AOT behaves as fractional Brownian motion with the Hurst exponent $H$.

\section{APPENDIX B}

\section{Fitting Structure Functions with Partial Saturation}

The real satellite data examples presented in Fig. 6 indicate that, in many cases, the structure function shapes deviate from the form described by Eqs. (12) and (16) and Fig. 1. The characteristic concave feature in the 100-500-km-scale range (partial saturation) suggests that these SFs are superpositions of two components corresponding to a trend(s) and a relatively stationary AOT field. While the trend component's SF is expected to be simply quadratic in scale, it appears that we can successfully fit the measured structure function using the same model for both components. This means that we formally assume that aerosol consists of two independent modes or layers. We need to keep in mind, however, that while the MBL SF has physical meaning, the representation of the trend contribution as a formal SF is an abstraction used only for fitting. Since parameters of the trend SF have no real meaning, we relax the requirement of $H<1$ for it to improve fitting flexibility.

We assume that the stationary and the trend components are statistically independent, as if they indeed correspond to two layers separated by height. Then the statistics of these components satisfy the system of equations following Eqs. (5)-(7):

$$
\begin{aligned}
S_{2}(r) & =S_{2}^{(1)}(r)+S_{2}^{(2)}(r), \\
\tau & =\tau_{1}+\tau_{2}, \\
s^{2} & =s_{1}^{2}+s_{2}^{2},
\end{aligned}
$$

where $\tau, s$, and $S_{2}(r)$ are known, while $\tau_{1}, \tau_{2}, s_{1}, s_{2}$, and the parameters of the two SF components are to be determined. Here and below, index 1 corresponds to the trend component, while index 2 corresponds to the MBL component. The retrieval algorithm is essentially a curve fitting of the measured $S_{2}(r)$ by the family of component SFs with parameters satisfying the conditions Eqs. (B2) and (B3). To make this fitting more robust and to reduce the number of retrieved parameters (which may have trade-offs between them), we 
complement the latter two equations with another condition:

$$
\frac{s_{1}}{\tau_{1}}=\frac{s_{2}}{\tau_{2}}
$$

(which is equivalent to $u_{1}=u_{2}$ ). We see in real satellite data shown in Fig. 7 that the ratio $s / \tau$ indeed is not very variable, so the assumption of Eq. (B4) is quite natural. In our approach, first the single-mode retrieval is performed to get an estimate $L_{e}$ of the variability scale. Then the fitting is performed over a single free parameter $\alpha$ that is the fraction of the trend component in the total variance. In this notation,

$$
s_{1}=s \sqrt{\alpha} \text { and } s_{2}=s \sqrt{1-\alpha},
$$

and the retrieval method utilizes the assumption that the MBL component's structure function $S^{(2)}(r)$ quickly saturates and is close to the constant $2 s_{2}^{2}$ in the scale range between $L_{e} / 2$ and $L_{e}$. Thus, for each value of $\alpha$ the trend SF in this range can be computed as

$$
S_{2}^{(1)}(r)=S_{2}(r)-2 s_{2}^{2}=S_{2}(r)-2 s^{2}(1-\alpha) .
$$

This SF is then fitted in the range $\left[L_{e} / 2, L_{e}\right]$ according to the method described in section 4 , given

$$
u_{1}=\frac{s_{1}^{2}}{\tau_{1}^{2}}+1=\frac{\left(s_{1}+s_{2}\right)^{2}}{\tau^{2}}+1=(\sqrt{\alpha}+\sqrt{1-\alpha})^{2} \frac{s^{2}}{\tau^{2}}+1 .
$$

Here, we used that according to Eq. (B4)

$$
\tau_{1}=\frac{s_{1}}{s_{1}+s_{2}} \tau .
$$

After the parameters of $S^{(1)}(r)$ are determined, its analytical form is derived from Eqs. (12) and (16) and subtracted from $S_{2}(r)$ to obtain $S_{2}^{(2)}(r)$, which is also parameterized using the single-mode method. For each value of the parameter $\alpha$ the tightness of the fit in the lag range $\left[0, L_{e}\right]$ of the measured structure function $S_{2}(r)$ by the corresponding analytical form $S_{2}^{(1)}(r)+S_{2}^{(2)}(r)$ is evaluated, and the value of $\alpha$ is determined by the best fit.

Figure B1 illustrates the above fitting method on the example of the data from the Indian Ocean, which is also presented in Fig. 6e. The red curve corresponds to the $\mathrm{SF}$ derived from the data. The partial saturation is clearly seen at the scales below $400 \mathrm{~km}$. The initial single-mode fit based on the variance observed in the sample is shown by the dashed blue curve. The discrepancy between the measured SF and the fit are evident, since the SF exhibits large-scale behavior

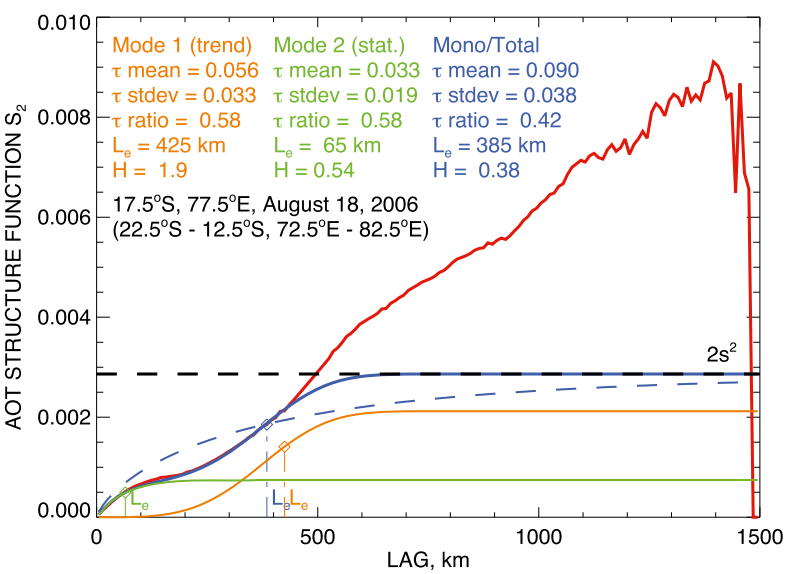

FIG. B1. Details of structure function fitting method. The data are from the middle of the Indian Ocean also presented in Fig. 6e. The red curve corresponds to the SF derived from the data, while the fits obtained from single-mode (dashed) and two-mode (solid) models are shown by blue lines. The SFs for the trend and stationary (boundary layer) AOT components from two-mode retrievals are shown by orange and green curves, respectively.

inconsistent with the local variance $s^{2}$ (the asymptote $2 s^{2}$ of the fitting curve is shown by the horizontal dashed line). The two-mode fit assuming the same variance $s^{2}$ is depicted by solid blue curve, while its trend and MBL components are represented in orange and green, respectively. This fit also significantly deviates from the measured SF at scales larger than $400 \mathrm{~km}$; however, it closely captures the SF's shape at smaller scales, allowing us to single out the MBL component's SF and to split the total AOT into within- and above-MBL parts.

\section{REFERENCES}

Alexandrov, M. D., A. Marshak, B. Cairns, A. A. Lacis, and B. E. Carlson, 2004: Scaling properties of aerosol optical thickness retrieved from ground-based measurements. J. Atmos. Sci., 61, 1024-1039, doi:10.1175/1520-0469(2004)061<1024: SPOAOT $>2.0 . \mathrm{CO} ; 2$.

Anderson, T. L., R. J. Charlson, D. M. Winker, J. A. Ogren, and K. Holmen, 2003: Mesoscale variations of tropospheric aerosols. J. Atmos. Sci., 60, 119-136, doi:10.1175/ 1520-0469(2003)060<0119:MVOTA > 2.0.CO;2.

Bell, T. L., 1987: A space-time stochastic model of rainfall for satellite remote-sensing studies. J. Geophys. Res., 92, 96319643, doi:10.1029/JD092iD08p09631.

Benedetti, A., and Coauthors, 2009: Aerosol analysis and forecast in the European Centre for Medium-Range Weather Forecasts Integrated Forecast System: 2. Data assimilation. J. Geophys. Res., 114, D13205, doi:10.1029/2008JD011115.

Cahalan, R. F., and J. B. Snider, 1989: Marine stratocumulus structure. Remote Sens. Environ., 28, 95-107, doi:10.1016/ 0034-4257(89)90108-9.

Curran, P. J., 1988: The semivariogram in remote sensing: An introduction. Remote Sens. Environ., 24, 493-507, doi:10.1016/ 0034-4257(88)90021-1. 
Davis, A., A. Marshak, W. Wiscombe, and R. Cahalan, 1994 Multifractal characterizations of nonstationarity and intermittency in geophysical fields: Observed, retrieved, or simulated. J. Geophys. Res., 99, 8055-8072, doi:10.1029/94JD00219.

,,$-- \ldots$, and $\longrightarrow, 1996$ : Scale invariance of liquid water distributions in marine stratocumulus. Part I: Spectral properties and stationarity issues. J. Atmos. Sci., 53, 1538-1558, doi:10.1175/1520-0469(1996)053<1538:SIOLWD>2.0.CO;2.

,$- \ldots$, R. Cahalan, and W. Wiscombe, 1997: The Landsat scale break in stratocumulus as a three-dimensional radiative transfer effect, implications for cloud remote sensing. J. Atmos. Sci., 54, 241-260, doi:10.1175/1520-0469(1997)054<0241: TLSBIS $>2.0 . \mathrm{CO} ; 2$.

,,-- H. Gerber, and W. Wiscombe, 1999: Horizontal structure of marine boundary layer clouds from centimeter to kilometer scales. J. Geophys. Res., 104, 6123-6144, doi:10.1029/ 1998JD200078.

Gage, K. S., and G. D. Nastrom, 1986: Theoretical interpretation of atmospheric wavenumber spectra of wind and temperature observed by commercial aircraft during GASP. J. Atmos. Sci., 43, 729-740, doi:10.1175/1520-0469(1986)043<0729: TIOAWS $>2.0 . \mathrm{CO} ; 2$.

Geogdzhayev, I., B. Cairns, M. Mishchenko, K. Tsigaridis, and T. van Noije, 2014: Model-based estimation of samplingcaused uncertainty in aerosol remote sensing for climate research applications. Quart. J. Roy. Meteor. Soc., 140, 2353-2363, doi:10.1002/qj.2305.

Hansen, J., M. Sato, R. Ruedy, A. Lacis, and V. Oinas, 2000: Global warming in the twenty-first century: An alternative scenario. Proc. Natl. Acad. Sci. USA, 97, 9875-9880, doi:10.1073/ pnas.170278997.

Huneeus, N., and Coauthors, 2011: Global dust model intercomparison in AeroCom phase I. Atmos. Chem. Phys., 11, 77817816, doi:10.5194/acp-11-7781-2011.

Kinne, S., and Coauthors, 2006: An AeroCom initial assessmentOptical properties in aerosol component modules of global models. Atmos. Chem. Phys., 6, 1815-1834, doi:10.5194/ acp-6-1815-2006.

Koch, D., T. C. Bond, D. Streets, N. Unger, and G. R. van der Werf, 2007: Global impacts of aerosols from particular source regions and sectors. J. Geophys. Res., 112, D02205, doi:10.1029/ 2005JD007024.

Kolmogorov, A. N., 1941: Local structure of turbulence in an incompressible liquid for very large Reynolds numbers. Dokl. Akad. Nauk SSSR, 30, 299-303.

Levy, R. C., L. A. Remer, R. G. Kleidman, S. Mattoo, C. Ichoku, R. Kahn, and T. F. Eck, 2010: Global evaluation of the Collection 5 MODIS dark-target aerosol products over land. Atmos. Chem. Phys., 10, 10399-10 420, doi:10.5194/acp-10-10399-2010.

Lilly, D., 1989: Two-dimensional turbulence generated by energy sources at two scales. J. Atmos. Sci., 46, 2026-2030, doi:10.1175/ 1520-0469(1989)046<2026:TDTGBE $>2.0$. CO;2.

Lovejoy, S., and D. Schertzer, 2010: Towards a new synthesis for atmospheric dynamics: Space-time cascades. Atmos. Res., 96, 1-52, doi:10.1016/j.atmosres.2010.01.004.

_ functions: Convenient choices for geophysics. Nonlinear Processes Geophys., 19, 513-527, doi:10.5194/npg-19-513-2012.

Mandelbrot, B. B., 1982: The Fractal Geometry of Nature. W. H. Freeman, $460 \mathrm{pp}$.

Marshak, A., A. Davis, W. Wiscombe, and R. Cahalan, 1997: Scale invariance of liquid water distributions in marine stratocumulus. Part II: Multifractal properties and intermittency issues. J. Atmos. Sci., 54, 1423-1444, doi:10.1175/ 1520-0469(1997)054<1423:SIILWD>2.0.CO;2.

Mejia, J. M., and I. Rodriguez-Iturbe, 1974: Correlation links between normal and log normal processes. Water Resour. Res., 10, 689-690, doi:10.1029/WR010i004p00689.

Morcrette, J.-J., and Coauthors, 2009: Aerosol analysis and forecast in the European Centre for Medium-Range Weather Forecasts Integrated Forecast System: Forward modeling. J. Geophys. Res., 114, D06206, doi:10.1029/ 2008JD011235.

Myhre, G., and Coauthors, 2013: Radiative forcing of the direct aerosol effect from AeroCom Phase II simulations. Atmos. Chem. Phys., 13, 1853-1877, doi:10.5194/acp-13-1853-2013.

O'Neill, N. T., A. Ignatov, B. N. Holben, and T. F. Eck, 2000: The lognormal distribution as a reference for reporting aerosol optical depth statistics; Empirical tests using multi-year, multisite AERONET Sunphotometer data. Geophys. Res. Lett., 27, 3333-3336, doi:10.1029/2000GL011581.

Quaas, J., and Coauthors, 2009: Aerosol indirect effects-General circulation model intercomparison and evaluation with satellite data. Atmos. Chem. Phys., 9, 8697-8717, doi:10.5194/ acp-9-8697-2009.

Redemann, J., M. A. Vaughan, Q. Zhang, Y. Shinozuka, P. B. Russell, J. M. Livingston, M. Kacenelenbogen, and L. A. Remer, 2012: The comparison of MODIS-Aqua (C5) and CALIOP (V2 \& V3) aerosol optical depth. Atmos. Chem. Phys., 12, 3025-3043, doi:10.5194/acp-12-3025-2012.

Remer, L. A., and Coauthors, 2005: The MODIS aerosol algorithm, products, and validation. J. Atmos. Sci., 62, 947-973, doi:10.1175/JAS3385.1.

_ and Coauthors, 2008: Global aerosol climatology from the MODIS satellite sensors. J. Geophys. Res., 113, D14S07, doi:10.1029/2007JD009661.

Schmidt, G. A., and Coauthors, 2014: Configuration and assessment of the GISS ModelE2 contributions to the CMIP5 archive. J. Adv. Model. Earth Syst., 6, 141-184, doi:10.1002/ 2013MS000265.

Takemura, T., H. Okamoto, Y. Maruyama, A. Numaguti, A. Higurashi, and T. Nakajima, 2000: Global three-dimensional simulation of aerosol optical thickness distribution of various origins. J. Geophys. Res., 105, 17853-17873, doi:10.1029/ 2000JD900265.

— - T. Nozawa, S. Emori, T. Y. Nakajima, and T. Nakajima, 2005: Simulation of climate response to aerosol direct and indirect effects with aerosol transport-radiation model. J. Geophys. Res., 110, D02202, doi:10.1029/2004JD005029.

Unger, N., D. T. Shindell, D. M. Koch, and D. G. Streets, 2008: Air pollution radiative forcing from specific emissions sectors at 2030. J. Geophys. Res., 113, D02306, doi:10.1029/ 2007JD008683.

Winker, D. M., M. A. Vaughan, A. H. Omar, Y. Hu, K. A. Powell, Z. Liu, W. H. Hunt, and S. A. Young, 2009: Overview of the CALIPSO mission and CALIOP data processing algorithms. J. Atmos. Oceanic Technol., 26, 2310-2323, doi:10.1175/ 2009JTECHA1281.1.

, and Coauthors, 2010: The CALIPSO mission: A global 3D view of aerosols and clouds. Bull. Amer. Meteor. Soc., 91, 1211-1229, doi:10.1175/2010BAMS3009.1.

Yao, M.-S., and Y. Cheng, 2012: Cloud simulations in response to turbulence parameterizations in the GISS model E GCM. J. Climate, 25, 4963-4974, doi:10.1175/JCLI-D-11-00399.1. 